\title{
Über das Schicksal des infundierten Zuckers mit besonderer Beriicksichtigung der Zuckeraufnahmefähigkeit der Gewebezellen.
}

\author{
II. Mitteilung. \\ Unterschied des Zuckergehaltes zwischen Arterien- und \\ Venenblut während und nach Glukosezufuhr. \\ Von \\ Dr. Toshio Kurokawa. \\ (黑 川 利 败) \\ (Aus der medizinischen Klinik ron Prof. S. Yamakawa \\ an der Universität zu Sendai.)
}

Es gab manche Autoren, welche aus dem Unterschied des Blutzuckergehaltes zwischen zu- und abführenden Gefässen auf den Zuckerverbrauch in den betreffenden Geweben und Organen schlossen und auf diese Weise bezüglich der Genese der Zuckerkrankheit oder des Wirkungsmechanismus des Insulins etwas Bestimmtes zu erkennen strebten. Schon früher teilte Bang ${ }^{1)}$ mit, dass ein Unterschied des Zuckergehaltes zwischen Arterienund Venenblut wirklich besteht. Nach ihm soll ein Mensch pro Tag etwa $700 \mathrm{~g}$ Kohlehydrat verbrauchen, und da in diesem Zeitverlauf ca. 7000 Liter Blut durch den Organismus zirkulieren, müssen die gesamten $700 \mathrm{~g}$ Glukose durch diese Menge Blut zu den Körperzellen transportiert werden. Folglich muss das Arterienblut etwa $0,01 \%$ mehr Zucker enthalten als das venöse; bei kleineren Tieren kann natürlich der Unterschied noch grösser sein. Auch die Untersuchungen von Lépine, ${ }^{2)} \mathrm{Cl}$. Bernard ${ }^{33}$ und Seegen ${ }^{4}$ zeigten, dass ein Unterschied des Blutzuckergehaltes zwischen Carotis und dem rechten Ventrikel oder zwischen V. hepatica und Portader besteht. Ausser diesen klassischen Autoren hatten sich auch mehrere an-

1) Bang, I., Der Blutzuciker, Wiesbaden 1913, 48.

2) Lépine, R., Le diabète sucre, Paris 1909, 69.

3) Cl. Bernard, Lécons sur le Diabète sucre, Paris 1877, 274.

4) J. Seegen, Pflügers Arch., 1885, 37, 348 u. 1886, 39, 121. 
dere Forscher mit diesem Problem beschäftigt, worauf ich noch später zurückkommen werde. Nachdem ich im vorigen Versuche durch Bestimmung des Gesamt-C des Blutes hatte bestätigen können, dass der grösste Teil des injizierten Zuckers sofort nach Infusion nicht mehr in irgendeiner Form im kreisenden Blut verweilt, und zur Vermutung gelangt war, dass der verschwundeue Zucker wahrscheinlich in den Geweben irgendwo verborgen sein müsse, möchte ich nun mit diesem und den nachfolgenden Versuchen weiter klarzustellen suchen, ob diese Auffassung zu Recht besteht oder nicht. Im vorliegenden Versuche wurde zuerst in verschiedenen Zeitabständen nach Zuckerzufuhr der Zuckerwert des Arterien- und entsprechenden Venenblutes gleichzeitig bestimmt, in der Absicht, aus dem Unterschied zwischen den beiden die Zuckeraufnahme in den Geweben des Stromgebietes anschaulich zu machen.

\section{Versuchsergebnisse.}

1. Versuche mit normalen Kaninchen.

Mittelgrosse Kaninchen, welche 24 Stunden vor dem Versuche gehungert hatten, wurden auf dem Operationsbrett in Rückenlage fixiert; nachdem man zuerst die beiderseitigen Femoralgefüsse, dann A. carotis sin. herauspräpariert, die letztere doppelt fest unterbunden und durchgeschnitten hatte, injizierte man den Versuchstieren $5 \mathrm{ccm} 50 \%$ iger Glukoselösung in den zentralen Stumpf der Carotis stromanfwärts. Die Infusion geschah im Laufe von ca. 2 Minuten und zwar in möglichst gleichmässiger Geschwindigkeit. In diesem Zeitverlauf traf das vom Herzen hinausgepumpte Blut im Aortenbogen mit der Zuckerlösung zusammen, und beide vermischten sich innig; ein Bruchteil des Blutes floss weiter durch Aorta abdominalis in die beiden Femoralarterien hinab, dann die Gewebe der hinteren Extremitäten und ging in venöses Blut über. Die Differenz des Blutzuckers in A. und V. femoralis, welche hierbei die in diesem Stromgebiete von den Geweben aufgenommene Zuckermenge darstellen muss, wurde in der Weise untersucht, dass während der Zuckerinfusion und den darauf folgenden 2 Stunden in kurzen Zeiträumen arterielles und venöses Blut gleichzeitig entnommen und auf seinen Zuckergehalt geprüft wurde. Dabei wurde arterielles Blut aus dem einen Schenkel und venöses aus dem anderen genommen. Der Grund, warum ich derart immer ungleichseitige Arterie und Vene verwendete, besteht darin, dass sonst wegen der Operation der Blutentnahme von einer Arterie die Zirkulation auf derselben Seite 
gestört und dadurch der natürliche Verlauf des Zuckergehaltes des venösen Blutes ein anderer werden kann. Die Blutzuckerbestimmung wurde nach Hagedorn'scher' Ferricyanidmethode ausgeführt. Was aber Blutentnahme und Enteiweissung betrifft, so bevorzugte ich hierbei die Bang'sche Fliesspapiermethode, weil es zwecks dieses Versuches unbedingt notwendig war, in kleinen Zeitintervallen möglichst häufig und schnell zu entbluten. Falls höherer Prozentgehalt des Blutzuckers den geeigneten Wert der Bestimmungsmethode übertraf, verdünnte ich das Filtrat zweckmässig mit Bang'scher Salzlösung.

Das Ergebnis der Versuche ist in beistehenden Figuren übersichtlich dargestellt. Aus den Abbildungen ist Folgendes hervorzuheben: Das Arterienblut war an Zuckergehalt schon normaliter ein bisschen höher als das venöse. Der Zuckergehalt des Arterien-

1) H.C. Hagedorn, u. B. N. Jensen, Biochem. Z., 1923, 135, 46 .

Abb. 1.

Arterieller und renöser Blutzucker während und nach Zuckerinfusion bei normalen Kaninchen.

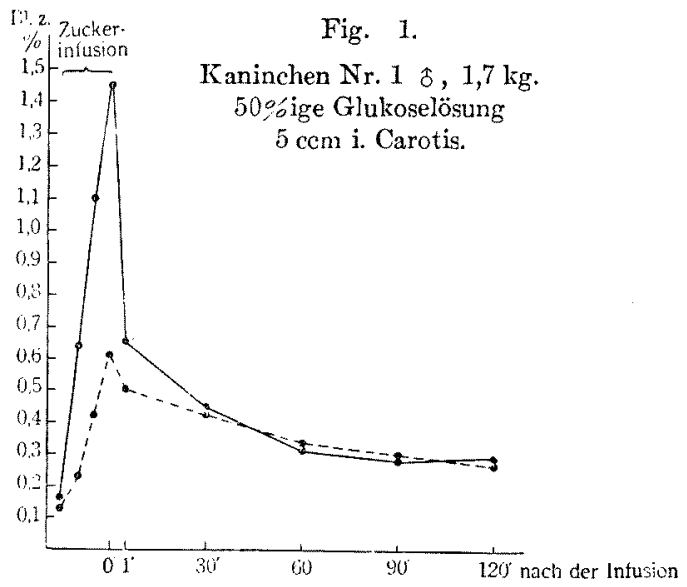

In sïmtlichen Figuren steht links die Schwankung des Blutzuckergehaltes während und sofort nach Infusion, wobei die Bestimmung in kurzen Zeitabständen hintereinander ausgeführt wurde, in Vergrösserung skizziert.

Ausgezogene Linie $=$ Arterieller Blutzucker.

Gestrichelte Linie $=$ Venöser Blutzucker.

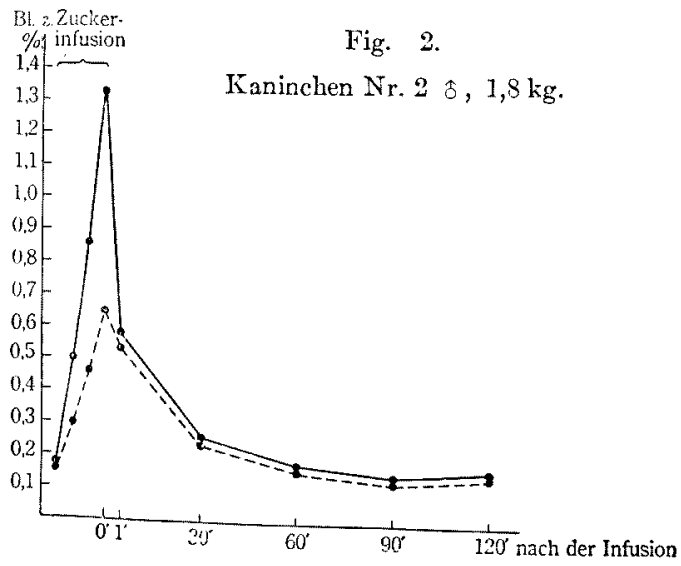



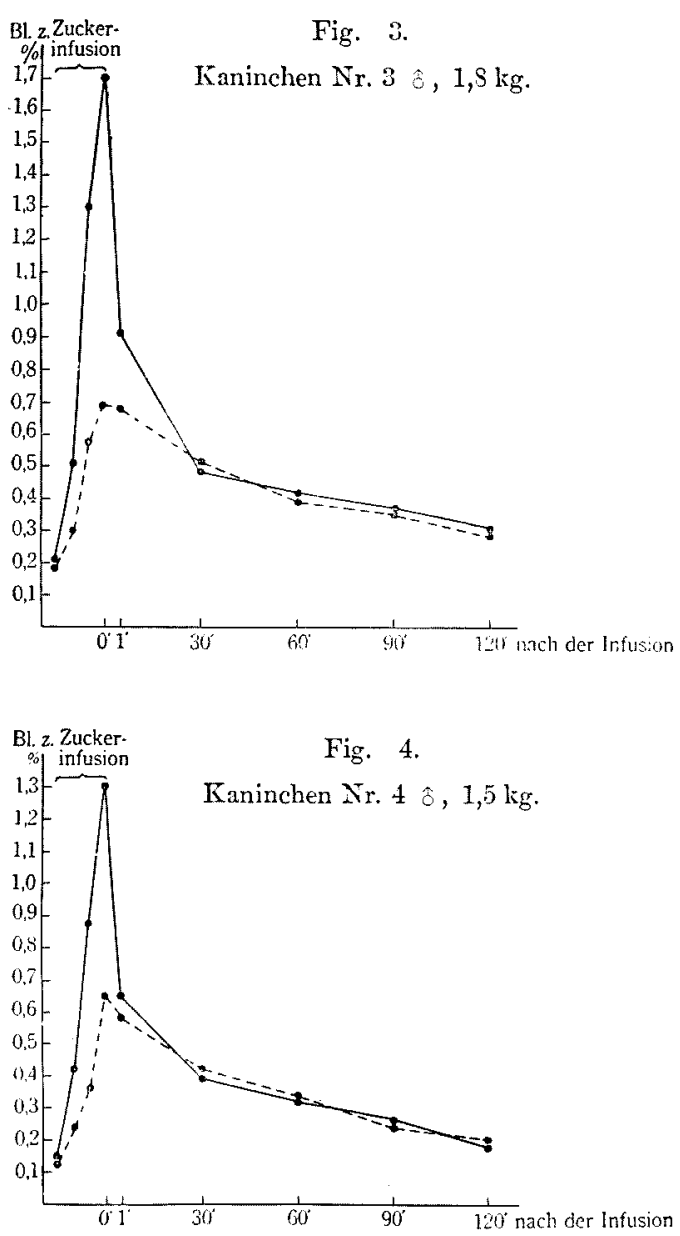

blutes stieg schon währénd der Zuckerinfusion plötzlich enorm hoch, und zwar bis $2 \%$ oder darüber, und 5 Minuten nach vollendeter Infusion sank er wieder rasch; dagegen zeigteder 'Zuckergehalt des in demselben Augenblicke entnommenen Venenblutes keine solch emporschnellende Schwankung, sondern stets eine viel trägere. Im weiteren Verlaufe rückte die arterielle Zuckerkurve allmählich der venösen immer nüher, wobei erstere bald etwas höher war als letztere, bald aber umgekehrt; nach 2 Stunden war schliesslich das ursprüngliche Verhältnis gewöhnlich wiederhergestellt. Dass in dieser Restitutions-

phase der Venenblutzucker bald einen höheren Wert als der arterielle zeigte, beruht sehr wahrscheinlich darauf, dass der übermüssig aufgenommene Zucker zum Teil aus dem Gewebe wieder ins Blut abgegeben wurde. An dieser Schwankung des Zuckergehaltes, besonders auf Seiten des venösen Blutes nahm natürlich auch die Flüssigkeitsverschiebung $z$ wischen Blut und Gewebe teil, die immer der Aufnahme und Abgabe des Zuckers entgegengesetzt vonstatten geht, wodurch das venöse Blut bald verdünnt, bald eingedickt wird. Jedenfalls ist wohl die hier beobach tete Differenz der beiden Zuckerkurven, der steilen arteriellen und der trägen venösen, in demselben Zeitraum nach Zuckerinfusion hauptsächlich als Folge der Zuckeraufnahme 
in den Geweben zu betrachten, wodurch der im Blute übermüssige Zucker schnell beseitigt wird.

Wie schon eingangs erörtert, haben die Gewebezellen schon physiologischerweise die Fähigkeit, aus strömendem Blut Zucker aufzunehmen, der weiter als Energiequelle zur Verfügung steht. Henrique und Ege $\mathrm{e}^{1 \text { ) }}$ gelang es, experimentell zu zeigen, dass strömendes Blut während der Passage im Muskel Zucker verliert. Cori, Cori und Goltz $z^{2}$ haben auch darauf hingewiesen, dass bei Menschen durchschnittlicl $8 \mathrm{mg} \%$ Differenz an Blutzuckergehalt zwischen $\mathrm{A}$. und V. femoralis besteht. Dieser positive Befund wurde auch von $\mathrm{Faber} \mathrm{r}^{3)}$ und Wertheimer ${ }^{4)}$ bei Menschen und von Frank und Nothmann ${ }^{5}$ bei Hunden bestïtigt. Es fehlt jedoch nicht an Autoren, die diese Differenz an Blutzuckergehalt überhaupt bestritten (Foster ${ }^{6)}$ und Lawrence ${ }^{7)}$ bei Menschen, Frank und Noth$\operatorname{mann}^{5}$ bei Kaninchen). Ob aber die Tatsache, dass ein so hoher Blutzuckergehalt, wie er in diesen Versuchen nach Infusion erscheint, der in physiologischen Zustande kaum in Betracht kommt, schnell wieder zur Norm hergestellt wird, auch auf diese physiologische Gewebsfunlition zurückzuführen sei, ist noch eine offene Frage. Der Organismus hat bekanntlich eine wunderbare biologische Akkommodationsfähigkeit, hartnäckig an seiner normalen Blutzusammensetzung festzuhalten. In jedem Notfalle, sei es ein spontaner oder ein künstlicher, der irgendeine fehlerhafte Blutzusammensetzung verursacht, die die ertrügliche Grenze gerade überschreitet, strebt er eifrigst, durch solche Abwehr sie wieder auszugleichen. Im Falle der Infusion einer hypertonischen Lösung des Zuckers, ebenso wie anderer blutfremder Substanzen, wird wahrscheinlich diese Vorrichtung auch ganz in Anspruch genommen. Alles in allem, konnte ich aus dem Ergebnis dieser Versuche indirekt bestätigen, dass nach Zufuhr hypertonischer Zuckerlösung der aus der Blutbahn verschwundene Zucker vorwiegend in die Gewebe aufgenommen wird.

1) V. Henrique, u. R. Ege, Biochem. Z., 1921, 119, 121. 21, 72.

2) Cori, C. F., G. T. Cori u. H. Goltz, Proc. of Soc. f. Exp. Biol. and Med., 1924,

3) Faber, K., Zit. nach „Insulin" von Laqueur, München_1925, 72.

4) M. Wertheimer, Kl. W., 1923, 2362.

5) E. Frank u. M. Nothmann, Kl. W., 1924, 581.

6) Foster, Zit. nach "Insulin" von Laqueur,"München 1925, 74.

7) Lawrence, R. D., Brit. Med. J., 1924, I, 516. 
2. Versuche mit pankreasdiabetischen Hunden ohne und unter Insulineinfluss.

Manche Autoren, die den Zuckergehalt des arteriellen und venösen Blutes diabetischer Menschen und Tiere vergleichend untersuchten, gaben an, dass dabei, im Gegensatz zu Stoffwechselgesunden, keine Differenz zwischen beiden besteht (Lawrence, ${ }^{1}$ Faber, ${ }^{2>}$ Wertheimer ${ }^{3)}$ ). Dieser Befund blieb aber nicht unwidersprochen, denn Cori $i^{4)}$ und Frank und Noth ma $\mathbf{n n}^{5)}$ beobachteten auch einen auderen Fall. Unter Insulineinfluss soll der gleichbleibende Zuckergehalt des arteriellen und venösen Blutes bei Diabetikern wieder voneinander abweichen (Law rence, F a ber), ${ }^{6)}$ was wieder von anderer Seite angefochten wurde (McCormick). ${ }^{7}$ ?

In einer früheren Abhandlung habe ich schon darauf hingewiesen, dass die Zuckeraufnahmef ähigkeit der Gewebe beim Diabetiker ebenso wie beim Normalen nicht gestört wird. Diese Annahme wurde aber dabei nicht auf direkte Analyse der Gewebe begründet, sondern bloss aus der

Abb. 2.

Arterieller und venöser Blutzucker während und nach

Zuckerinfusion bei normalen Hunden.

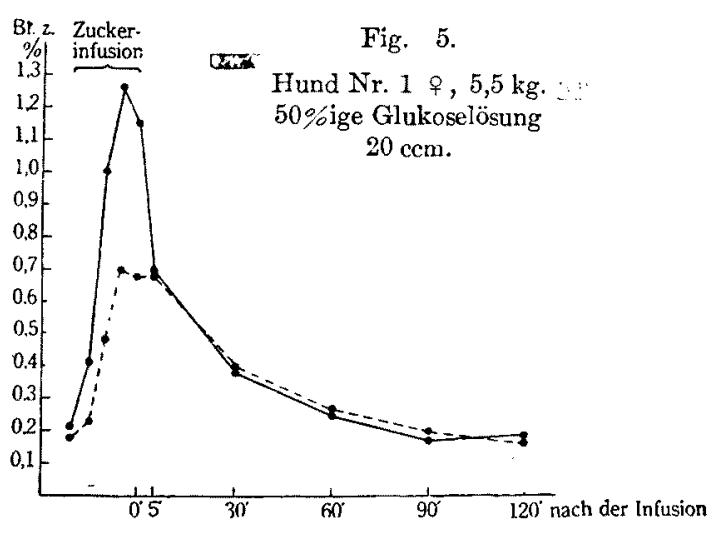

1) Lawrence, R. D., Brit. Med., J., 1924, 520.

2) Faber, K., zit. nach "Insulin" ron Laque ur, München 1925, 74.

3) M. Wertheimer, Kl. W., 1923, 2362.

4) Cori, C. F., Proc. of Soc. f. Exp. Biol. and Med., 1924, $21,72$.

5) Frank, E. u. Nothmann, M., Kl. W., 1924, 582.

6) Lawrence, R. D., u. Faber, K., Zit. nach „Insulin" ron Laqueur, München 1925, 74 .

7) M c Cormick, N. A., Am. J. Physiol., 1924, 68, 144. 
Boobachtung der Blutzuckerkurve gefolgert, die sich nach Zufuhr hypertonischer Zuckerlösung bei beiden als fast gleich erwies. Jetzt will ich diese Frage bei dieser Gelegenheit wieder aufnehmen, um durch Untersuchung der Zuckerverluste während der Zirkulation durch die Gewebe meiner früheren Auffassung eine festere Grundlage zu geben.

$\mathrm{Zu}$ dieser Untersuchung standen auch wie beim früheren Versuche pankreasdiabetische und als Kontrolle stoffwechselgesunde Hunde zur Verfügung. Gleichzeitig wurde auch der Insulineinfluss auf die Zuckeraufnahmef:ihigkeit der Gewebe beobachtet. Den Tieren wurden $20 \mathrm{ccm}$ $50 \%$ iger Zuckerlösung in A.carotissin. stromanfwärts langsam im Verlaufe von a. 4 Minuten eingespritzt. Die Versuchsanordnung gestaltete sich im allgemeinen gleich wie beim vorigen Kaninchenversuche.

Bii normalen Hunden stieg, wie aus Kurve $2 \mathrm{zu}$ ersehen ist, die Blutzuckerkurve des arteriellen Blutes nach Zuckerinfusion emporschnellend hoch hinauf,

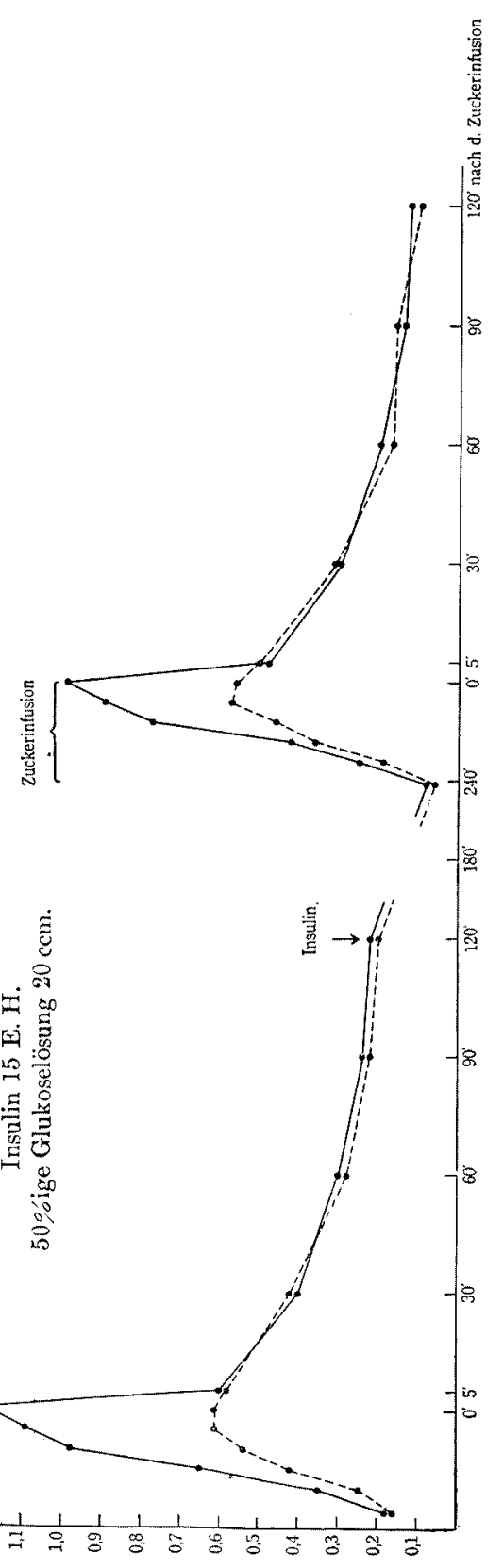


die des venösen jedoch nur träg; also der beim Kaninchenversuch erhobene Befund wurde hier wieder bestätigt. Bei demselben Hunde, der vorher zur Hypoglykïmie insulinisiert und dem die gleiche Menge von Glukoselösung intravenös injiziert worden, war der maximale Blutzuckergehalt in allgemeinen etwas geringer als ohne Insulin, aber der Unterschied des Zuckergehaltes zwischen Arterien- und Venenblut bot trotz des merklichen Insulineinflusses keine nachweisbare Abweichung; also der Befund von Cori und Frank und Nothmann, dass durch Insulin im Zuckergehalt des arteriellen und venösen Blutes eine weitgehende Differenz hervorgerufen wird, wurde hier bei Zuckerinfusion nicht bestätigt. Daraus folgt, dass beim Stoffwechselgesunden In-

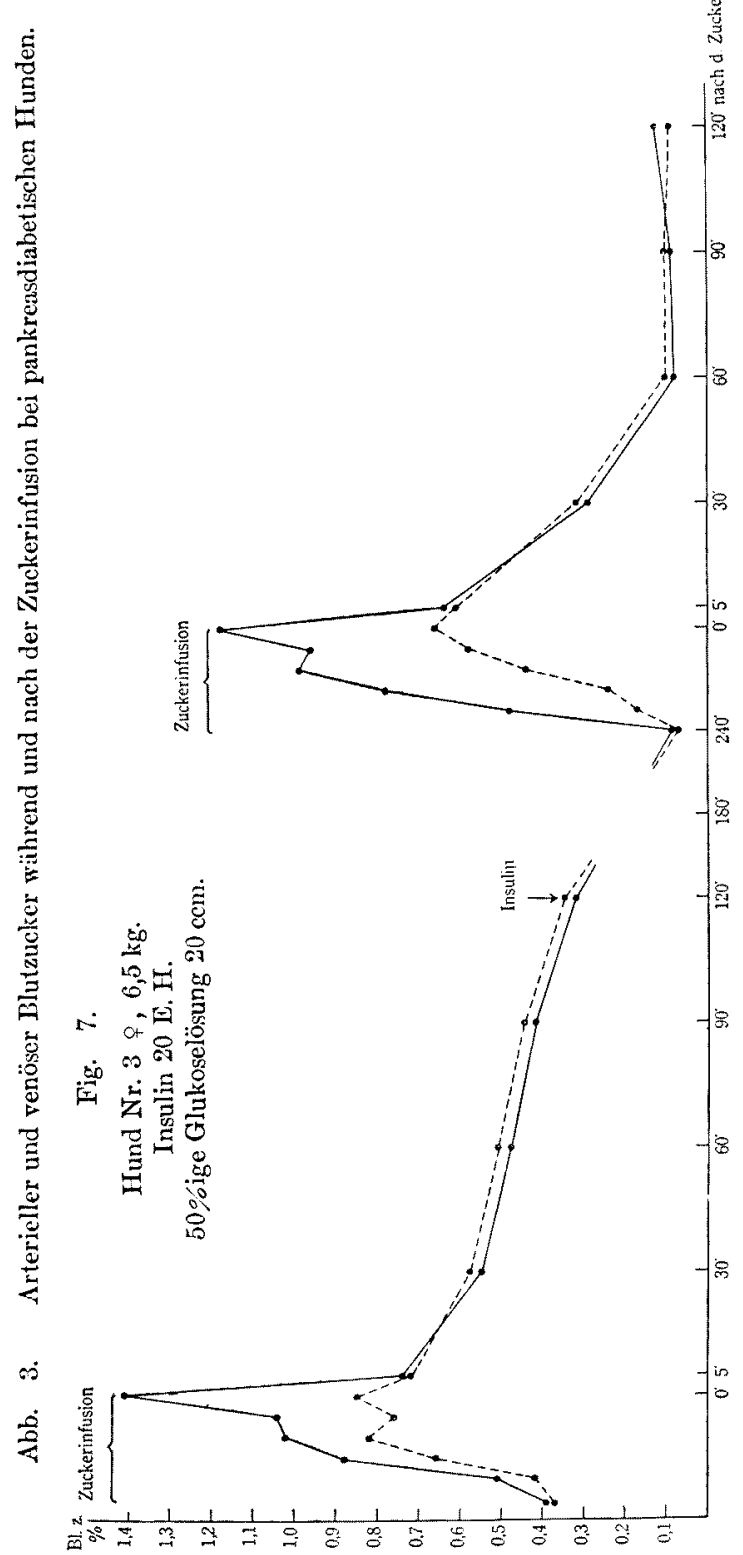




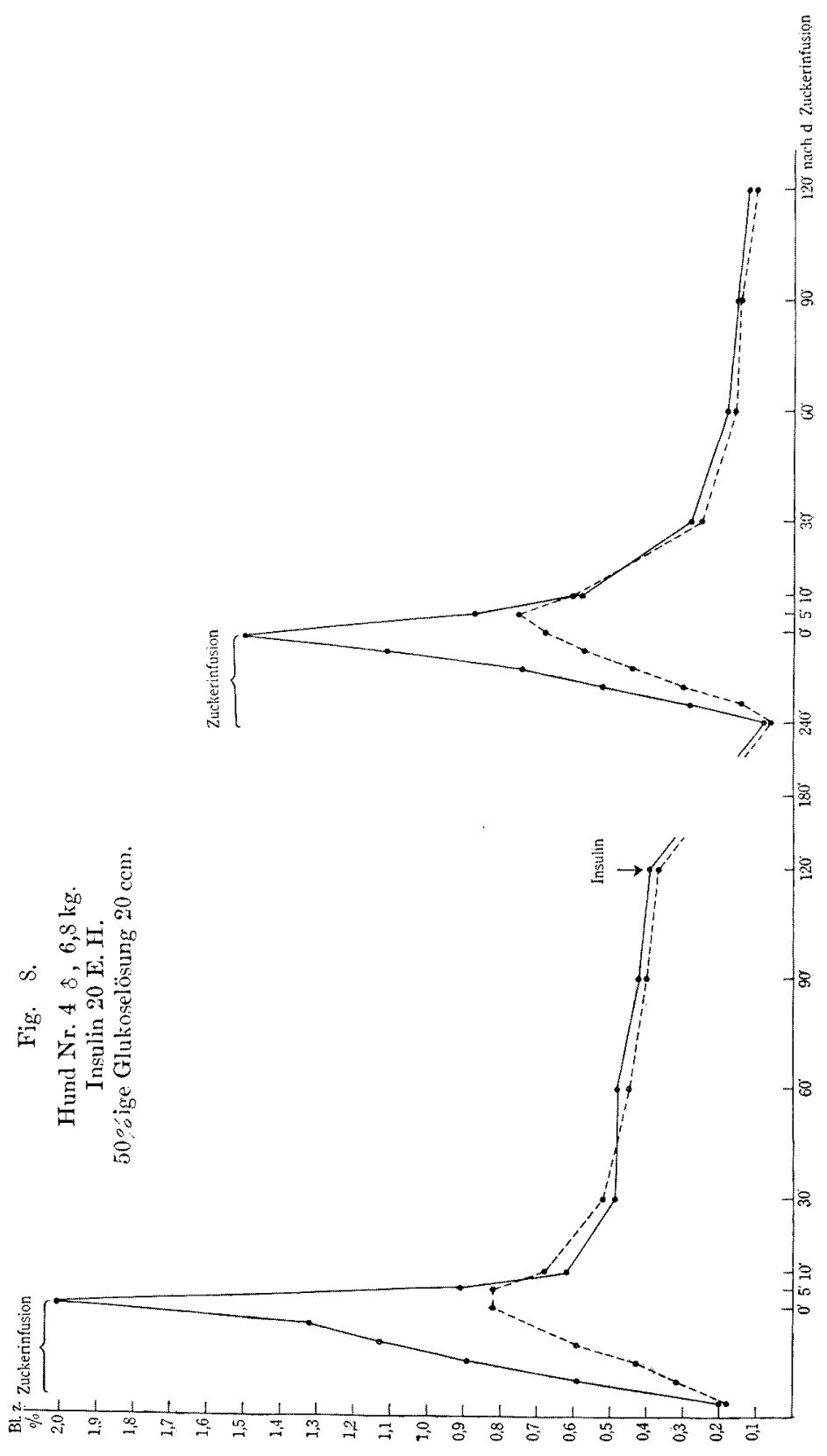




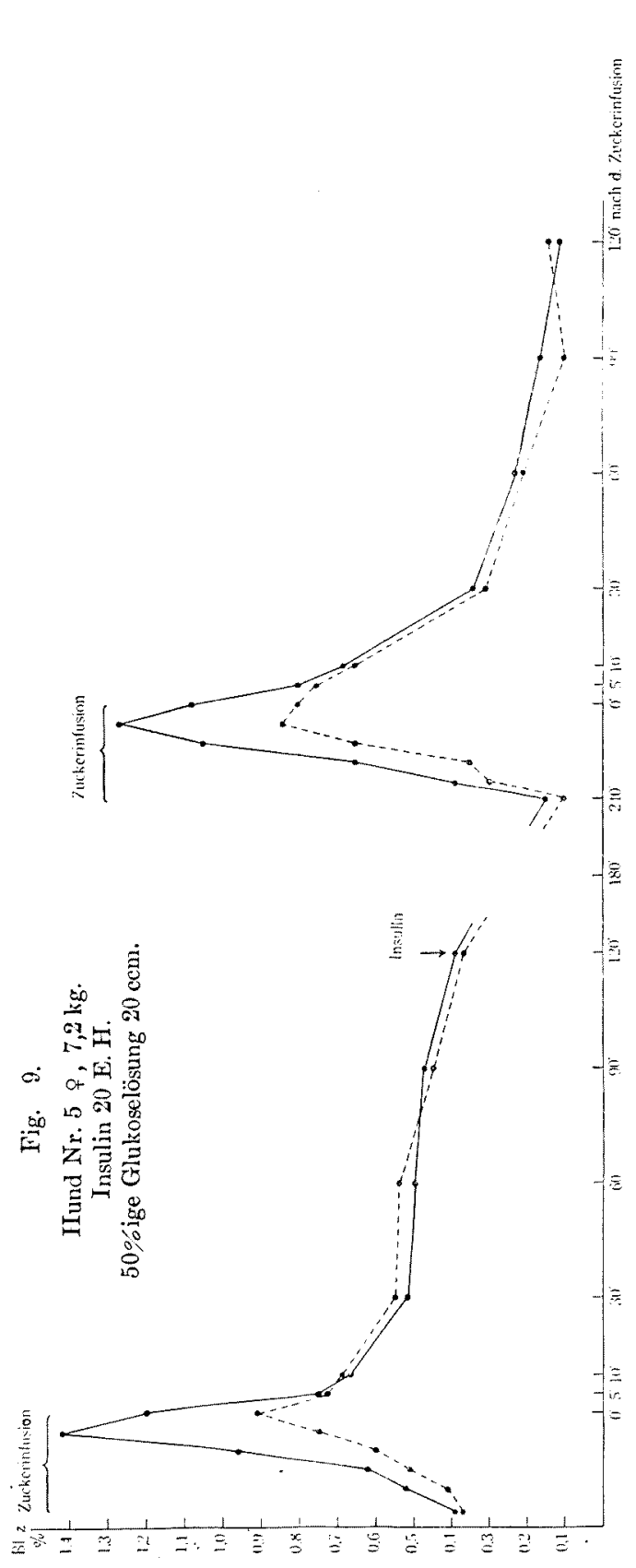

sulin auf die Aufnahmefïhigkeit intravenös injizierten Zuckers keinen nennenswerten Einfluss ausübt, worauf ich schon in einer früheren $\mathrm{Ab}$ handlung ${ }^{1)}$ ausdrücklich hingewiesen habe.

Die Kurve 3 gibt die Resultate des Versuches mit pankreasdiabetischen Hunden wieder. Vor Glukosezufuhr war der Zuckergehalt des arteriellen Blutes mit dem des venösen fast gleich oder sogar manchmal ein bisschen tiefer als letzterer; ein Befund, der die Angabe von Lawrence, Faber und Wertheimer bestätigte. Wührend und direkt nach Zuckerzufuhr ïberschritt ersterer weitaus letzteren, aber der Unterschied zwischen beiden zeigte auch hierbei keine merkbare Abweichung von dem Befund bei Stoffwechselgesunden. In der

1) Kurokawa, T., Tohoku Journ. Exp. Med., $1926,8,66$. 
Mehrzahl der Fälle übertraf der Zuckergehalt des venösen Blutes im Anfang der Restitutionsphase den des arteriellen ein bisschen, was aber niemals für Diabetesfälle charakteristisch war, denn es wurde auch bei Normalen ebenso häufig beobachtet.

Im insulinisierten Zustande verhielt sich der Blutzuckerverlauf der diabetischen Hunde nach Zuckerinfusion fast gleich wie beim Fall ohne Insulin, bis auf die Restitutionsphase, bei der der Zuckergehalt sowohl des arteriellen als auch des venösen Blutes viel schneller sank als bei letzterem, was aus der bekannten Insulinwirkung leicht verständlich ist.

\section{Zusammenfassung.}

(1) Nach intravenöser Infusion von hypertonischer Glukoselösung vermehrt sich der Zuckergehalt des Arterienblutes schnell bedentend, im Gegensatz dazu der des venösen nur träge. Diese Differenz der Blutzuckerkurve beruht darauf, dass der infundierte Zucker während der Passage durch die kapilläre Strombahn reichlich von den Geweben aufgenommen wird.

(2) Die Zuckeraufnahmefähigkeit der Gewebezellen, die beim Stoffwechselgesunden beobachtet wird, bleibt auch bei Diabetikern ungestört erhalten.

(3) Insulin übt auf die Zuckeraufnahme der Gewebezellen keinen besonderen Einfluss aus. 